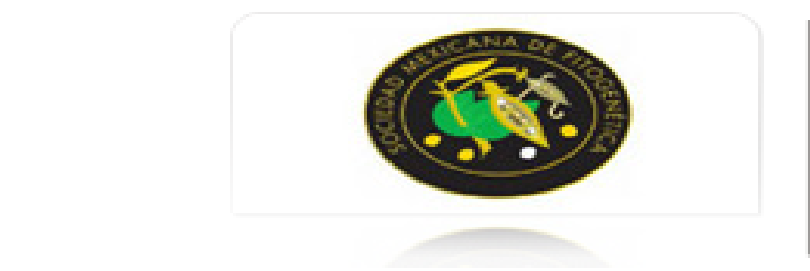

Revista Fitotecnia Mexicana

ISSN: 0187-7380

revfitotecniamex@gmail.com

Sociedad Mexicana de Fitogenética, A.C.

México

Peña-Lomelí, Aureliano; Ponce-Valerio, Juan J.; Sánchez-del-Castillo, Felipe; Magaña-Lira, Natanael DESEMPEÑO AGRONÓMICO DE VARIEDADES DE TOMATE DE CÁSCARA EN INVERNADERO Y CAMPO ABIERTO

Revista Fitotecnia Mexicana, vol. 37, núm. 4, 2014, pp. 381-391

Sociedad Mexicana de Fitogenética, A.C.

Chapingo, México

Disponible en: http://www.redalyc.org/articulo.oa?id=61032672011

Cómo citar el artículo

- Número completo

- Más información del artículo

Página de la revista en redalyc.org

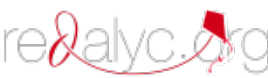

Sistema de Información Científica

Red de Revistas Científicas de América Latina, el Caribe, España y Portugal Proyecto académico sin fines de lucro, desarrollado bajo la iniciativa de acceso abierto 


\title{
DESEMPEÑO AGRONÓMICO DE VARIEDADES DE TOMATE DE CÁSCARA EN INVERNADERO Y CAMPO ABIERTO
}

\section{AGRONOMIC PERFORMANCE OF HUSK TOMATO VARIETIES UNDER GREENHOUSE AND OPEN FIELD CONDITIONS}

\author{
Aureliano Peña-Lomelíi, Juan J. Ponce-Valerio² ${ }^{2}$ Felipe Sánchez-del-Castillo ${ }^{1}$ \\ y Natanael Magaña-Lira ${ }^{3}$
}

\begin{abstract}
${ }^{1}$ Departamento de Fitotecnia, Universidad Autónoma Chapingo. Km 38.5 Carr. México-Texcoco. 56230, Chapingo, Edo. de México, México. Tel.-Fax: (595) 952-1642. ${ }^{2}$ Gerente Técnico. Desarrollos Tecnificados Agrícolas S. A. de C. V. Km 0.5 Carr. San Ignacio-Magdalena. 54160, Magdalena, Sonora, México. ${ }^{3}$ Campo Experimental Valle de México, Instituto Nacional de Investigaciones Forestales, Agrícolas y Pecuarias. Km 13.5 Carr. Los Reyes-Texcoco. 56250, Coatlinchán, Edo. de México, México.
\end{abstract}

${ }^{*}$ Autor para correspondencia (aplomeli@correo.chapingo.mx )

\section{RESUMEN}

Se estudió la respuesta productiva de 40 variedades de tomate de cáscara (Physalis ixocarpa Brot. ex Horm.) en cuatro sistemas de producción (riego rodado, riego por goteo con y sin acolchado plástico e hidroponía bajo invernadero), con el objetivo de determinar las mejores variedades para cada sistema y comparar la producción entre sistemas. La investigación se llevó a cabo en Chapingo, Estado de México, durante el ciclo primavera-verano 2003, bajo un diseño experimental de bloques completos al azar con tres repeticiones. El mejor sistema de producción fue a campo abierto con riego por goteo y acolchado plástico, tanto en rendimiento ( $948 \mathrm{~g} / \mathrm{planta}$ ) como en tamaño de fruto ( $31 \mathrm{~g} /$ fruto), aunque en este último rasgo no superó al sistema de hidroponía. En promedio de los cuatro sistemas de producción, las variedades de mayor rendimiento fueron Población 3 (1102 g/planta), Población 5 (1025 g/planta), CHF1-Chapingo (989 g/ planta) y Manzano SM2R (972 g/planta), con frutos de tamaño mediano. Las variedades de mayor tamaño de fruto fueron Puebla SM2 (47 g/ fruto), Puebla SM1 (46 g/fruto), San Mateo (45 g/fruto) y Mahune (45 $\mathrm{g} /$ fruto), pero presentaron bajo rendimiento. Las variedades de mayor rendimiento son de la raza Rendidora, y las de mayor tamaño de fruto de la raza Puebla. La variedad Diamante resultó de fruto grande (41 g/fruto) y rendimiento superior a la media ( $787 \mathrm{~g} /$ planta). Para cada sistema de producción, las variedades de mayor rendimiento fueron Población 3, Población 5, Población 3 y 124 USA 03, respectivamente; en tanto que las de mayor tamaño de fruto fueron Puebla SM1, San Mateo, Puebla SM2 y Mahune, respectivamente.

Palabras clave: Physalis ixocarpa, sistemas de producción, variedades mejoradas.

\section{SUMMARY}

This research determined yield of 40 husk tomato (Physalis ixocarpa Brot. ex Horm.) varieties under four production systems (ditch irrigation, drip irrigation, with or without plastic cover, and hydroponics under greenhouse), to determine the best variety for each production system, and to compare production among systems. The trial was conducted at Chapingo, Estado de México during the 2003 Spring-Summer season using a completely randomized block experimental design with three replications. The best production system was drip irrigation with plastic cover, which yielded $948 \mathrm{~g} / \mathrm{plant}$ and $31 \mathrm{~g} /$ fruit; however, fruit size was not improved on this hydroponic system. On average out of the four production systems, the varieties with best yield were Population 3 (1102 g/plant), Population 5 (1025 g/plant), CHF1-Chapingo (989 g/plant), and Manzano SM2R (972 g/ plant), with medium fruit size. Varieties with the largest fruit sizes were Puebla SM2 (47 g/fruit), Puebla SM1 (46 g/fruit), San Mateo (45 g/fruit) and Mahune (45 g/fruit), although they had low yield. The varieties with the highest yield were from Rendidora race, and the ones with the largest fruit size were from Puebla race. Diamante variety produced large fruits $(41 \mathrm{~g} /$ fruit) and yield $(787 \mathrm{~g} / \mathrm{plant})$ above the average. For each production system, the varieties with higher yield were Population 3, Population 5, Population 3, and 124 USA 03, respectively; while for fruit size, those with the largest fruit were Puebla SM1, San Mateo, Puebla SM2, and Mahune, respectively.

Index words: Physalis ixocarpa, production systems, improved varieties.

\section{INTRODUCCIÓN}

El tomate de cáscara (Physalis ixocarpa Brot. ex Horm.), conocido también como tomate verde, tomatillo o tomate de fresadilla, es una hortaliza cuyo uso más difundido es el alimenticio. Se cultiva en 28 de los 32 estados de la República Mexicana. En el año 2010 se sembraron 48,475 ha, lo que ubica a esta especie en el quinto lugar en superficie cultivada con respecto a las demás hortalizas. De la superficie cultivada $73.4 \%$ es bajo riego, principalmente en los estados de Sinaloa, Sonora, Puebla, Guanajuato, Michoacán y Zacatecas; el resto (26.6 \%) se siembra bajo condiciones de temporal (secano), donde sobresalen los estados de Jalisco, Estado de México, Sinaloa, Morelos, Nayarit y Puebla. Se cultiva tanto en el ciclo primavera-verano (46.5\%) como en el de otoño-invierno (SIAP-SAGARPA, 2010).

El cultivo de tomate de cáscara se ha incrementado debido a que es una hortaliza que no requiere muchos cuidados. No obstante, el rendimiento promedio nacional es bajo en relación con el potencial productivo del cultivo, que se estima en 40 t ha $^{-1}$ (Peña y Santiaguillo, 1999). Tal rendimiento es posible con el uso de variedades mejoradas y técnicas 
adecuadas de cultivo. Además, tiene grandes perspectivas en el mercado, donde incluso puede ser un sustituto del tomate (Solanum lycopersicon L.), pues se cotiza a buen precio y en ocasiones superior al de éste (SNIIM, 2012). Tiene la ventaja de que su ciclo de producción es relativamente corto $(80 \mathrm{~d})$ ya que su fruto se consume cuando aún se encuentra fisiológicamente inmaduro (Cruz-Álvarez et al., 2012). El consumo per capita nacional es alto $(3.5 \mathrm{~kg})$ y su exportación a Estados Unidos de América y Canadá es cada vez mayor (Peña y Santiaguillo, 1999).

La dinámica agrícola del cultivo del tomate de cáscara demanda la generación de cultivares mejorados que se ajusten a las necesidades actuales del mercado nacional e internacional. Entre las características a mejorar destacan el rendimiento, hábito de crecimiento, distribución de la producción, así como color, forma y tamaño de fruto. El concentrar la producción en un tiempo reducido debe ser unos de los objetivos del mejoramiento genético de la especie, al menos en regiones en donde las bajas temperaturas son limitantes para su siembra, como el altiplano mexicano, ya que esto junto con la precocidad permitiría llegar al mercado más pronto y reduciría los costos de recolección (Peña y Márquez, 1990; Peña et al., 2008).

Actualmente existen 11 variedades (CHF1-Chapingo, Rendidora, Diamante, Tamazula SM3, Milpero Tetela, Yema de Huevo, Manzano Tepetlixpa, Puebla SM3, Tecozautla 04, Salamanca y Potrero) descritas con la guía de la Unión para la Protección de Obtenciones Vegetales (UPOV) y registradas en México (Peña et al., 2011) ante el Servicio Nacional de Inspección y Certificación de Semillas (SNICS), pero el proceso de producción se basa principalmente en poblaciones nativas que los propios productores conservan más otras que las compañías productoras de semilla incrementan y comercializan. En este contexto, es necesario generar, evaluar y registrar tanto variedades nativas como mejoradas, para ponerlas a disposición de los productores y contribuir con ello al incremento del rendimiento del cultivo y a la conservación del material genético (Peña et al., 2008; Peña et al., 2011).

La expresión del potencial de rendimiento de los cultivos depende del genotipo, el ambiente y la interacción genotipo $\mathrm{x}$ ambiente. Es por ello importante estudiar simultáneamente diferentes variedades en cada sistema de producción, para determinar cuál es la mejor variedad dentro de cada uno de ellos. En tomate de cáscara, el sistema de producción de riego rodado es el más frecuente; no obstante, ya existen áreas a nivel comercial donde se utiliza riego por goteo con o sin acolchado plástico, bajo condiciones de cielo abierto (Peña et al., 2008).

Si bien se está desarrollando investigación en relación con su cultivo en hidroponía bajo invernadero (Castro et al., 2000), aún no se ha reportado el comportamiento de variedades de tomate de cáscara bajo los diferentes sistemas de producción convencionales o emergentes. Con base en lo anterior, en el presente trabajo se evaluó la respuesta productiva de 40 variedades en cuatro sistemas de producción: a) riego rodado, b) riego por goteo sin acolchado, c) riego por goteo con acolchado plástico, y d) hidroponía bajo invernadero, para determinar las mejores variedades para cada sistema y en promedio de éstos, con base en rendimiento y tamaño de fruto.

\section{MATERIALES Y MÉTODOS}

La investigación se llevó a cabo en un campo experimental con suelo franco-arenoso-arcilloso (Cachón, 1974), ubicado en Chapingo, Estado de México, durante el ciclo primavera-verano 2003. Se evaluaron 40 variedades, de las cuales 14 son comerciales, 18 son producto del programa de mejoramiento genético de tomate de cáscara de la Universidad Autónoma Chapingo (UACh) y ocho son materiales del Banco Nacional de Germoplasma de la UACh (Cuadro 3). Las 40 variedades se establecieron en cuatro sistemas de producción. De éstos, tres fueron en campo abierto (riego rodado, riego por goteo sin acolchado y riego por goteo con acolchado plástico) y uno en invernadero con hidroponía.

En campo abierto la densidad de población fue de 20,750 plantas/ha. La unidad experimental constó de 16 plantas establecidas en un surco de $1.2 \mathrm{~m}$ de ancho, $6 \mathrm{~m}$ de largo y $0.4 \mathrm{~m}$ de distancia entre plantas. La fertilización se realizó con la fórmula 200-100-100 de N- $\mathrm{P}_{2} \mathrm{O}_{5}-\mathrm{KO}_{2}$, respectivamente. Los riegos se aplicaron cuando el suelo tenía entre 15 y 20 cbar de tensión de humedad, según registros con un potenciómetro-tensiómetro portátil Kelway ${ }^{\circledR}$ modelo MA78 (United Kingdom).

En hidroponía la densidad fue de 20,000 plantas/ha de invernadero ( $80 \%$ de superficie útil). La unidad experimental fue de seis plantas distribuidas en un una hilera de $0.8 \mathrm{~m}$ de ancho, $2.5 \mathrm{~m}$ de largo y distancia entre plantas de $0.5 \mathrm{~m}$. Como sustrato se utilizó arena volcánica roja, localmente conocida como tezontle. Se utilizó la solución nutritiva recomendada por Pérez y Castro (1999), y los riegos fueron diarios.

En cada sistema de producción los tratamientos se distribuyeron en un diseño experimental de bloques completos al azar con tres repeticiones. Debido a que el tomate de cáscara es una especie gaméticamente autoincompatible, en el invernadero se colocó una colmena de abejas (Apis mellifera) para facilitar la polinización cruzada. Las características evaluadas en tres cortes de fruto fueron las siguientes: peso por fruto (PPF, en g), como un indicador del tamaño de 
fruto, registrado en una muestra de 10 frutos por unidad experimental; y rendimiento por planta (RPP, en g), determinado mediante la división del rendimiento entre el número de plantas por unidad experimental. El peso promedio por fruto (PPPF) se estimó con el promedio de los valores de PPF en los tres cortes. Se calculó el rendimiento total por planta (RTP) mediante la suma de RPP en los tres cortes.

Los análisis de varianza se hicieron con base en el modelo fijo de una serie de experimentos (cuatro sistemas de producción) en bloques completos al azar y la comparación de medias con la prueba de Tukey (Sahagún, 1993). Dado que la interacción fue significativa entre sistemas de producción y variedades, también se realizó un análisis individual por sistema de producción con el propósito de identificar a las variedades con mejor comportamiento en cada sistema.

\section{RESULTADOS Y DISCUSIÓN}

Las variables peso por fruto (PPF) y rendimiento por planta (RPP) en cada uno de los tres cortes y en promedio (PPPF) y total (RTP) (Cuadro 1), presentan diferencias significativas $(\mathrm{P} \leq 0.05)$ entre los sistemas de producción y entre variedades, con interacción significativa entre dichos factores, excepto para PPF en el primer corte. La interacción indica que algunas variedades tuvieron comportamiento diferente en cada sistema de producción, y que en cada uno de éstos la mejor variedad es diferente. Los coeficientes de variación fueron particularmente altos en rendimiento por corte, situación atribuida principalmente a variaciones en el criterio de cosecha de los obreros y a la variabilidad genética de la especie, aspecto común en tomate de cáscara (Peña et al., 2008; Santiaguillo et al., 2012).

\section{Comparación de sistemas de producción}

En peso por fruto en el primer corte (Cuadro 2), el sistema de riego por goteo con acolchado superó $(\mathrm{P} \leq 0.05)$ a los otros tres sistemas, y el riego por goteo sin acolchado presentó el menor peso por fruto, sin diferencia significativa entre riego rodado e hidroponía bajo invernadero. En peso promedio por fruto a través de cortes (PPPF), los sistemas de goteo con acolchado e hidroponía bajo invernadero fueron estadísticamente iguales y ambos superaron $(\mathrm{P} \leq 0.05)$ a los otros dos sistemas, de los cuales riego por goteo sin acolchado produjo el menor PPPF. Este mismo comportamiento se observó en el segundo corte, mientras que en el tercero la hidroponía bajo invernadero superó a los otros tres sistemas (Cuadro 2). Se puede inferir que el mayor tamaño de fruto se obtiene con los sistemas de hidroponía bajo invernadero y de riego por goteo con acolchado, que según Castro et al. (2000) ocurre cuando hay mejores condiciones para el desarrollo de la planta.

Para rendimiento por planta el sistema de riego por goteo con acolchado fue el mejor, ya que superó $(\mathrm{P} \leq 0.05)$ a los tres sistemas restantes (Cuadro 2), excepto al de hidroponía

Cuadro 1. Cuadrados medios del análisis de varianza combinado de cuatro sistemas de producción y 40 variedades de tomate de cáscara evaluadas en Chapingo, México, para peso por fruto (g) por corte y en promedio de cortes (PPPF), y para rendimiento por planta (g) por corte y en total de cortes (RTP).

\begin{tabular}{|c|c|c|c|c|c|}
\hline Fuentes de variación & GL & PPFC1 & PPFC2 & PPFC3 & PPPF \\
\hline Sistema de producción & 3 & $8533^{*}$ & $874^{*}$ & $3281^{*}$ & $2774^{*}$ \\
\hline Repetición (Sistema) & 8 & $754^{*}$ & $146^{*}$ & $174^{*}$ & $205^{*}$ \\
\hline Variedad & 39 & $1565 *$ & $801 *$ & $728^{*}$ & $952 *$ \\
\hline Sistema x Variedad & 117 & 165 & $81^{*}$ & $37^{*}$ & $44^{*}$ \\
\hline Error & 312 & 130 & 51 & 26 & 32 \\
\hline \multirow[t]{2}{*}{ CV (\%) } & & 37 & 26 & 21 & 21 \\
\hline & & RPPC1 & RPPC2 & RPPC3 & RTP \\
\hline Sistema de producción & 3 & $691,847^{*}$ & $447,207^{*}$ & $1,280,772 *$ & $5,425,011^{*}$ \\
\hline Repetición (Sistema) & 8 & $37749^{*}$ & 11,628 & $102,416^{*}$ & $229,107^{*}$ \\
\hline Variedad & 39 & $194,238^{*}$ & $99,828^{*}$ & 25,522 * & $484,997^{\star}$ \\
\hline $\begin{array}{l}\text { Sistema x Variedad } \\
\text { Error }\end{array}$ & $\begin{array}{l}117 \\
312\end{array}$ & $\begin{array}{l}19,454^{*} \\
14,181\end{array}$ & $\begin{array}{l}22,506^{*} \\
15,820\end{array}$ & $\begin{array}{l}27,814 \text { * } \\
14,516\end{array}$ & $\begin{array}{l}97,006^{*} \\
42,958\end{array}$ \\
\hline CV (\%) & & 65 & 48 & 61 & 32 \\
\hline
\end{tabular}

GL = grados de libertad; PPFC1, PPFC2 y PPFC3 = peso por fruto en el corte uno, dos y tres, respectivamente. RPPC1, RPPC2 y RPPC3 = rendimiento por planta en el corte uno, dos y tres, respectivamente. $\mathrm{CV}=$ coeficiente de variación. ${ }^{\star}$ Significancia con $\mathrm{P} \leq 0.05$. 
en el segundo corte. Este resultado concuerda con el obtenido por Soldevilla et al. (2002), quienes compararon riego por goteo con y sin acolchado plástico en tomate de cáscara. Lo anterior pone de manifiesto la bondad del acolchado en hacer un uso más eficiente del agua y los nutrientes por la planta, incrementar la temperatura del suelo, y reducir la presencia de maleza y el ataque de patógenos (Csizirtszky et al., 1995).

En el lote donde se establecieron estos experimentos visualmente se detectó un ataque severo de barrenadores del tallo (Trichobaris championi Barber y Melanagromyza tomaterae Steyskal) y del hongo Fusarium, cuya incidencia fue menor cuando se utilizó acolchado; por ello convendría hacer una investigación posterior para evaluar la incidencia de plagas y enfermedades. El sistema de hidroponía bajo invernadero presentó un menor rendimiento en el primer corte así como en rendimiento total por planta $(\mathrm{P} \leq 0.05)$ con respecto al riego por goteo con acolchado (Cuadro 2). Esto pudo deberse a una falta de polinización, ya que el tomate de cáscara es una especie alógama obligada (Peña y Márquez, 1990), que en las primeras etapas de desarrollo de la planta tiene un bajo número de flores, aspecto que no propicia la concurrencia de polinizadores, entre ellos las abejas, ni la dispersión de polen por falta de movimiento de aire, ya que en marzo y abril el invernadero permaneció cerrado por más horas para contrarrestar las bajas temperaturas durante las primeras y últimas horas del día.

Por ello es necesario introducir al invernadero a otros insectos polinizadores como abejorros, o propiciar la dispersión de polen con ventiladores. Al parecer, las abejas que se colocaron dentro del invernadero no fueron suficientes durante este periodo, porque cuando había pocas flores tendían a salir del invernadero o se morían, sobre todo si la temperatura era superior a $40^{\circ} \mathrm{C}$. Por esto, en tanto no se resuelva el problema de polinización no es recomendable producir tomate de cáscara bajo invernadero, ya que si ésta no ocurre el rendimiento puede reducirse a cero.
La inferioridad de rendimiento en el sistema riego por goteo sin acolchar respecto al de acolchado concuerda con lo reportado por Soldevilla et al. (2002) para tomate de cáscara. En resumen, el sistema de riego por goteo con acolchado fue el mejor por presentar los mayores valores de rendimiento por planta y tamaño de fruto.

\section{Comparación de variedades en promedio de sistemas de producción}

En el Cuadro 3 se observa que la variedad Puebla SM2 tuvo frutos de mayor tamaño (PPPF) que otras 34 variedades, pero fue estadísticamente igual $(\mathrm{P} \leq 0.05)$ a Puebla SM1, Mahune, San Mateo, Tecamachalco y Diamante. Este grupo superior de variedades representa a las de mayor peso promedio por fruto; por ejemplo, el híbrido interracial Diamante superó a 31 variedades en dicha característi$\mathrm{ca}$, atribuible a que uno de sus progenitores es la variedad Tecamachalco de la raza Puebla (Santiaguillo et al., 2004). Las variedades de la raza Puebla destacaron por formar los frutos de mayor tamaño, lo cual coincide con lo reportado por Peña et al. (1999).

En el primer corte la variedad que tuvo mayor tamaño de fruto fue Puebla SM2, que superó $(\mathrm{P} \leq 0.05)$ a 29 variedades, y fue estadísticamente igual a diez variedades que en su mayoría también son variedades de la raza Puebla (Cuadro 3 ). En promedio de las variedades, los frutos del primer corte fueron los de mayor peso (PPFC1), que luego disminuyó su expresión en los cortes subsecuentes, lo cual es un comportamiento normal en tomate de cáscara (Pérez et al., 1997; Peña et al., 2008).

En el Cuadro 4 se puede observar que para rendimiento total (RTP) el grupo superior $(\mathrm{P} \leq 0.05)$ estuvo constituido por 12 variedades, entre las cuales no hubo diferencias. Con excepción de las variedades Manzano SM2 y Puebla verde, el resto son de la raza Rendidora o cruzas con ésta (Boyeros y Diamante), por lo que se puede corrobora que Rendidora es la raza más rendidora, como

Cuadro 2. Comparación de cuatro sistemas de producción de tomate de cáscara, en peso por fruto (PPF) y rendimiento por planta (RPP) por corte y para el promedio (PPPF) y total (RTP) de cortes, en promedio de 40 variedades.

\begin{tabular}{|c|c|c|c|c|c|c|c|c|}
\hline \multirow{2}{*}{ Sistema de producción } & \multicolumn{4}{|c|}{ PPF } & \multicolumn{4}{|c|}{ RPP } \\
\hline & Corte 1 & Corte 2 & Corte 3 & PPPF & Corte 1 & Corte 2 & Corte 3 & RTP \\
\hline Riego por goteo con acolchado & $41.4 \mathrm{a}^{\dagger}$ & $29.6 \mathrm{a}$ & $23.2 \mathrm{~b}$ & $31.4 \mathrm{a}$ & $292.4 \mathrm{a}$ & $313.7 \mathrm{a}$ & $341.8 \mathrm{a}$ & $947.8 \mathrm{a}$ \\
\hline Hidroponía bajo invernadero & $32.5 \mathrm{~b}$ & $30.0 \mathrm{a}$ & $31.0 \mathrm{a}$ & $31.2 \mathrm{a}$ & $116.9 c$ & $296.8 \mathrm{a}$ & $201.9 \mathrm{~b}$ & $615.5 b$ \\
\hline Riego rodado & $30.0 \mathrm{~b}$ & $26.9 \mathrm{~b}$ & $22.1 \mathrm{~b}$ & $26.4 \mathrm{~b}$ & $163.2 \mathrm{~b}$ & $254.3 \mathrm{~b}$ & $147.5 \mathrm{c}$ & $607.7 \mathrm{~b}$ \\
\hline Riego por goteo sin acolchado & $20.9 c$ & $24.2 \mathrm{c}$ & $18.7 \mathrm{c}$ & $21.2 \mathrm{c}$ & $159.2 \mathrm{~b}$ & $176.9 \mathrm{c}$ & $104.3 \mathrm{~d}$ & $440.3 \mathrm{c}$ \\
\hline DMSH & 3.8 & 2.4 & 1.7 & 1.9 & 39.7 & 41.9 & 40.2 & 69.1 \\
\hline
\end{tabular}

${ }^{\dagger}$ Medias con la misma letra en una columna no difieren estadísticamente (Tukey, 0.05); DMSH = diferencia mínima significativa honesta. 
Cuadro 3. Comparación de 40 variedades de tomate de cáscara crecidas en Chapingo, Estado de México, en peso por fruto (g) por corte y en promedio de cortes (PPPF), en promedio de cuatro sistemas de producción.

\begin{tabular}{|c|c|c|c|c|c|}
\hline Variedad & Raza & Corte 1 & Corte 2 & Corte 3 & PPPF \\
\hline 1. CHF1-Chapingo & R1 & $33.9 \mathrm{~d}-\mathrm{n}^{\dagger}$ & $26.6 \mathrm{f}-\mathrm{j}$ & 20.8 i-n & $27.0 \mathrm{e}-\mathrm{i}$ \\
\hline 2. Sintético interfamiliar & $\mathrm{R} 1$ & $25.6 \mathrm{~h}-\mathrm{n}$ & 28.8 e-i & $23.1 \mathrm{~h}-\mathrm{j}$ & 25.9 e-k \\
\hline 3. Familia 130 & R1 & $36.5 \mathrm{~b}-\mathrm{k}$ & $26.3 \mathrm{f}-\mathrm{j}$ & $19.8 \mathrm{i}-\mathrm{n}$ & $27.5 \mathrm{e}-\mathrm{i}$ \\
\hline 4. Población 8 & R1 & $34.7 \mathrm{~d}-\mathrm{m}$ & $30.9 \mathrm{c}-\mathrm{h}$ & $23.7 \mathrm{~g}-\mathrm{j}$ & $29.8 \mathrm{~d}-\mathrm{h}$ \\
\hline 5. Población 5 & $\mathrm{R} 1$ & $35.6 \mathrm{c}-1$ & 29.3 e-i & 22.9 h-k & $29.3 \mathrm{~d}-\mathrm{h}$ \\
\hline 6. Población 3 & $\mathrm{R} 1$ & $30.3 \mathrm{f}-\mathrm{n}$ & $26.6 f-j$ & $23.9 \mathrm{f}-\mathrm{j}$ & $26.9 e-j$ \\
\hline 7. Población 2 & R1 & $31.4 \mathrm{f}-\mathrm{n}$ & 30.2 d-h & $25.6 \mathrm{e}-\mathrm{j}$ & $29.0 \mathrm{~d}-\mathrm{h}$ \\
\hline 8. Sintético intervarietal & I1 & $44.6 \mathrm{a}-\mathrm{g}$ & $35.6 \mathrm{~b}-\mathrm{g}$ & $31.8 \mathrm{c}-\mathrm{g}$ & $37.3 \mathrm{~b}-\mathrm{d}$ \\
\hline 9. Manzano SM2R & M1 & 33.6 d-n & $32.5 \mathrm{~b}-\mathrm{h}$ & 27.4 e-i & $31.2 \mathrm{~d}-\mathrm{g}$ \\
\hline 10. Puebla SM1 & P1 & 50.2 a-e & $42.8 \mathrm{ab}$ & $43.7 \mathrm{a}$ & $45.6 \mathrm{ab}$ \\
\hline 11. Esmeralda & $\mathrm{R} 2$ & $21.9 \mathrm{j}-\mathrm{n}$ & $23.8 \mathrm{~h}-\mathrm{j}$ & $21.1 \mathrm{i}-\mathrm{n}$ & $22.3 \mathrm{f}-\mathrm{n}$ \\
\hline 12. Verde 3000 & P2 & $17.2 \mathrm{mn}$ & $22.1 \mathrm{~h}-\mathrm{j}$ & $19.2 \mathrm{j}-\mathrm{n}$ & $19.5 \mathrm{i}-\mathrm{n}$ \\
\hline 13. Verde supremo & $\mathrm{R} 2$ & $22.3 \mathrm{j}-\mathrm{n}$ & $24.5 \mathrm{~g}-\mathrm{j}$ & $21.8 \mathrm{i}-\mathrm{m}$ & $22.8 \mathrm{f}-\mathrm{n}$ \\
\hline 14. Yoreme & $\mathrm{T} 2$ & $21.8 \mathrm{j}-\mathrm{n}$ & $22.9 \mathrm{~h}-\mathrm{j}$ & 20.4 i-n & 21.7 g-n \\
\hline 15. Súper morado & $\mathrm{T} 2$ & $22.5 \mathrm{j}-\mathrm{n}$ & $17.0 \mathrm{j}$ & $14.41-\mathrm{n}$ & $18.0 \mathrm{j}-\mathrm{n}$ \\
\hline 16. Súper cerro gordo & S2 & $17.81-\mathrm{n}$ & $16.4 \mathrm{j}$ & $13.0 \mathrm{n}$ & $15.7 \mathrm{mn}$ \\
\hline 17. Tamazula SM2 & $\mathrm{T} 1$ & $17.61-n$ & $16.4 \mathrm{j}$ & $13.7 \mathrm{mn}$ & $15.9 \mathrm{mn}$ \\
\hline 18. San Mateo & $\mathrm{P} 1$ & $54.0 \mathrm{ab}$ & $40.1 \mathrm{a}-\mathrm{e}$ & $39.5 \mathrm{a}-\mathrm{c}$ & $44.6 \mathrm{ab}$ \\
\hline 19. Diamante & I1 & 50.7 a-d & 39.2 a-e & $31.9 \mathrm{c}-\mathrm{f}$ & 40.6 a-c \\
\hline 20. Puebla SM2 & P1 & $55.0 \mathrm{a}$ & $49.7 \mathrm{a}$ & $36.5 \mathrm{a}-\mathrm{d}$ & $47.1 \mathrm{a}$ \\
\hline 21. Tamazula SM2R & $\mathrm{T} 1$ & $20.4 \mathrm{j}-\mathrm{n}$ & $17.0 \mathrm{j}$ & $13.1 \mathrm{n}$ & $16.8 \mathrm{k}-\mathrm{n}$ \\
\hline 22. Tamazula SM3 & $\mathrm{T} 1$ & $17.71-n$ & $18.2 \mathrm{ij}$ & $13.8 \mathrm{mn}$ & $16.61-n$ \\
\hline 23. 19 JAL.74 & $\mathrm{T} 3$ & $18.3 \mathrm{k}-\mathrm{n}$ & $17.2 \mathrm{j}$ & $14.9 \mathrm{k}-\mathrm{n}$ & $16.8 \mathrm{l}-\mathrm{n}$ \\
\hline 24. 185 JAL. 68 & $\mathrm{~T} 3$ & $16.1 \mathrm{n}$ & $15.7 \mathrm{j}$ & $13.7 \mathrm{mn}$ & $15.2 \mathrm{n}$ \\
\hline 25. 12 GUSA02 & R3 & $21.5 \mathrm{j}-\mathrm{n}$ & $22.5 \mathrm{~h}-\mathrm{j}$ & $18.3 \mathrm{j}-\mathrm{n}$ & 20.8 h-n \\
\hline 26. Puebla verde & P2 & 31.9 e-n & $26.6 \mathrm{f}-\mathrm{j}$ & $22.4 \mathrm{~h}-1$ & $26.9 \mathrm{e}-\mathrm{j}$ \\
\hline 27. 128 BCN01ELSA & R3 & 36.9 a-j & $26.6 \mathrm{f}-\mathrm{j}$ & $24.8 \mathrm{e}-\mathrm{j}$ & 29.4 d-h \\
\hline 28. 124 USA 03 & R3 & $34.6 \mathrm{~d}-\mathrm{m}$ & $25.6 \mathrm{~g}-\mathrm{j}$ & 20.8 i-n & 27.0 e-i \\
\hline 29. 127 USA 03 & R3 & $27.7 \mathrm{f}-\mathrm{n}$ & $24.4 \mathrm{~g}-\mathrm{j}$ & 20.0 i-n & $24.0 \mathrm{f}-\mathrm{n}$ \\
\hline 30. $129 \mathrm{BCN} 02$ & R3 & 39.4 a-i & $29.5 \mathrm{e}-\mathrm{i}$ & $22.6 \mathrm{~h}-\mathrm{k}$ & $30.5 \mathrm{~d}-\mathrm{g}$ \\
\hline 31. Mahune & P2 & $53.7 \mathrm{a}-\mathrm{c}$ & $41.1 \mathrm{a}-\mathrm{d}$ & $40.3 \mathrm{ab}$ & $45.0 \mathrm{ab}$ \\
\hline 32. Cerro gordo & S2 & $25.7 \mathrm{~h}-\mathrm{n}$ & $26.5 \mathrm{f}-\mathrm{j}$ & $20.5 \mathrm{i}-\mathrm{n}$ & $24.2 \mathrm{f}-\mathrm{m}$ \\
\hline 33. San Román & $\mathrm{P} 4$ & $26.5 \mathrm{~g}-\mathrm{n}$ & $26.0 \mathrm{f}-\mathrm{j}$ & $23.8 \mathrm{f}-\mathrm{j}$ & 25.4 e-1 \\
\hline 34. Rendidora mejorada & $\mathrm{R} 2$ & $22.5 \mathrm{j}-\mathrm{n}$ & $24.5 \mathrm{~g}-\mathrm{j}$ & $22.6 \mathrm{~h}-\mathrm{k}$ & $23.2 \mathrm{f}-\mathrm{n}$ \\
\hline 35. Salamanca & S2 & $23.8 \mathrm{j}-\mathrm{n}$ & $26.8 \mathrm{f}-\mathrm{j}$ & $22.9 \mathrm{~h}-\mathrm{k}$ & $24.3 \mathrm{f}-\mathrm{m}$ \\
\hline 36. Cerro gordo AN & S4 & 25.1 h-n & $25.1 \mathrm{~g}-\mathrm{j}$ & $22.4 \mathrm{~h}-\mathrm{kl}$ & $24.2 \mathrm{f}-\mathrm{m}$ \\
\hline 37. Boyeros & I1 & $42.3 \mathrm{a}-\mathrm{h}$ & $37.3 \mathrm{~b}-\mathrm{f}$ & $32.5 \mathrm{~b}-\mathrm{e}$ & $37.3 \mathrm{~b}-\mathrm{d}$ \\
\hline 38. Tecamachalco & P1 & $45.3 \mathrm{a}-\mathrm{f}$ & $41.8 \mathrm{a}-\mathrm{c}$ & 36.6 a-d & $41.3 \mathrm{a}-\mathrm{c}$ \\
\hline 39. Divino & S2 & $38.4 a-j$ & $31.2 \mathrm{c}-\mathrm{h}$ & $30.5 \mathrm{~d}-\mathrm{h}$ & $33.4 \mathrm{c}-\mathrm{e}$ \\
\hline 40. 126 USA 02 & R3 & $23.1 \mathrm{j}-\mathrm{n}$ & $21.9 \mathrm{~h}-\mathrm{j}$ & $20.2 \mathrm{i}-\mathrm{n}$ & $24.7 \mathrm{~g}-\mathrm{n}$ \\
\hline DMSH & & 18.3 & 11.4 & 8.1 & 9.1 \\
\hline Promedio & & 31.2 & 27.7 & 23.8 & 27.5 \\
\hline
\end{tabular}

$\mathrm{R}=$ Rendidora; $\mathrm{I}$ = Híbrido Interracial; $\mathrm{M}$ = Manzano; $\mathrm{P}$ = Puebla; $\mathrm{T}$ = Tamazula; $\mathrm{S}$ = Salamanca; 1 = Programa de Mejoramiento Genético UACh; 2 = Material de Compañías Semilleras; 3 = Banco de Germoplasma UACh; 4 = Universidad Autónoma Agraria Antonio Narro; DMSH = diferencia mínima significativa honesta. ${ }^{\dagger}$ Medias con la misma letra en una columna no difieren estadísticamente (Tukey, 0.05); sólo se incluyen las letras inicial y final de cada media unidas por un guión. 
antes ya lo habían propuesto Peña et al. (1999).

De las 40 variedades evaluadas, las cuatro más rendidoras fueron Población 3, Población 5, CHF1-Chapingo y Manzano SM2R. Las Poblaciones 3 y 5 fueron derivadas de la variedad CHF1-Chapingo de la raza Rendidora, mientras que la otra es de la raza Manzano. De estas cuatro variedades, la Población 3 superó $(\mathrm{P} \leq 0.05)$ a 28 variedades; la Población 5, a 23; la CHF1-Chapingo, a 21; y la Manzano SM2R, a 19. Lo anterior indica que son materiales promisorios como variedades comerciales. De hecho, la variedad CHF1-Chapingo ya fue registrada por el SNICS y se ha desempeñado con éxito en campos de producción comercial. Por su parte, la variedad Diamante destacó como una de las más rendidoras (787 g/planta) y de fruto grande (41 g/fruto).

En el primer corte (Cuadro 4) las variedades de mayor rendimiento fueron Población 3, CHF1-Chapingo, Población 5 y Población 8, todas de la raza Rendidora, sin diferencia significativa entre ellas. La Población 3 superó a 36 variedades; la CHF1-Chapingo, a 31; la Población 5, a 27, y la Población 8, a 23 variedades. Sólo las Poblaciones 3 y 5, y la variedad CHF1-Chapingo se ubicaron en el grupo "mejor $10 \%$ " de las 40 variedades evaluadas. En el segundo corte las variedades Manzano SM2R, Población 3, Población 5 y Población 2 se ubicaron entre las mejores 20 variedades. La Población 3 sobresalió en los dos primeros cortes, lo que explica el porqué fue la mejor variedad en rendimiento total.

En cambio, las variedades de menor rendimiento total en general presentaron bajo rendimiento en el primer corte y el más alto en el último ( 15 de las 21 variedades con RTP menor a la media general), lo cual indica que son tardías (Cuadro 4). Se corrobora así que el rendimiento en el primer corte es fundamental para obtener un alto rendimiento total, como antes fue reportado por Peña et al. (2008) quienes además encontraron que la correlación genética aditiva fue la más alta entre 12 características.

El análisis conjunto del PPPF y RTP (Cuadros 3 y 4) permite señalar que las cuatro variedades de mayor rendimiento total por planta presentaron frutos de tamaño mediano, pues su PPPF fue similar a la media general para dicha característica. En contraste, las cuatro variedades de mayor PPPF presentaron un RTP menor a la media general y también menor rendimiento en el primer corte que en los siguientes dos, lo cual indica que dichas variedades son tardías.

Con base en lo anterior, se puede decir que la precocidad está asociada con alto RTP y fruto mediano, y que ciclo tardío está relacionado con frutos grandes y bajo rendimiento, como lo propusieron Peña et al. (2008) basados en las correlaciones genéticas entre estos rasgos. No obstante, es posible que las variedades tardías no hayan expresado todo su potencial de rendimiento, tal vez por problemas fitosanitarios.

Destacan tres variedades (Boyeros, Diamante y Sintético intervarietal) que presentaron un rendimiento total superior a la media general (ocuparon el $6^{\circ}, 10^{\circ}$ y $11^{\circ}$ lugar, respectivamente) y fruto grande (en $8^{\circ}, 6^{\circ}$ y $7^{\circ}$ lugar, respectivamente), que provienen de cruzamientos interraciales entre las variedades CHF1-Chapingo y Tecamachalco, la primera altamente rendidora y la segunda de fruto grande (Cuadros 3 y 4). Estos híbridos interraciales presentaron, en promedio de cada raza, el mayor PPPF y ocuparon el segundo lugar en RTP, superados solamente por la única variedad evaluada de la raza Manzano con dos ciclos de selección masal.

\section{Comparación de variedades en cada sistema de producción}

Para el sistema de producción de riego rodado las variedades de mayor peso promedio por fruto fueron Puebla SM1, Mahune, San Mateo, Tecamachalco, Puebla SM2, Boyeros y Sintético Intervarietal, sin diferencia significativa entre ellas (Cuadro 5), aunque sólo la primera y la segunda superaron $(\mathrm{P} \leq 0.05)$ a 32 variedades y la tercera a 26 . Las variedades Boyeros, Diamante, Mahune y Tecamachalco ubicadas en el grupo de mayor PPPF, también se ubicaron en el grupo superior de variedades con mayor RTP (Cuadro 6).

En el sistema de producción de riego por goteo sin acolchado las variedades Puebla SM2 y Puebla SM1 fueron las de mayor peso (Cuadro 5). La primera superó a 34 variedades y la segunda a 31; la variedad Diamante ocupó el tercer lugar y la Mahune el cuarto, donde la primera superó a nueve variedades y la segunda a siete. Para el sistema de producción de riego por goteo con acolchado, las variedades que sobresalieron en PPPF fueron San Mateo, Puebla SM2, Tecamachalco, Mahune y Puebla SM1, que superaron a 35, $31,31,30$ y 26 variedades, respectivamente. Estas cinco variedades son de la raza Puebla, lo que confirma que ésta es de frutos de tamaño grande. Para el sistema de producción de hidroponía bajo invernadero, si bien 23 variedades fueron estadísticamente iguales en PPPF, cuatro de ellas (Mahune, Puebla SM2, San Mateo y Puebla SM1) constituyeron el $10 \%$ superior para esta variable, todas ellas con germoplasma de la raza Puebla.

En cuanto al rendimiento total por planta (RTP), para el sistema de producción de riego rodado las variedades de mayor rendimiento de fruto fueron Población 3, Población 8 , Población 5 y Boyeros, y la primera superó a 23 variedades 
Cuadro 4. Medias de rendimiento por planta (g) por corte y en total de cortes (RTP) de 40 variedades de tomate de cáscara evaluadas en Chapingo, México, en promedio de cuatro sistemas de producción.

\begin{tabular}{|c|c|c|c|c|}
\hline Variedad & Corte 1 & Corte 2 & Corte 3 & RTP \\
\hline 1. CHF1-Chapingo & $456.5 \mathrm{ab}^{\dagger}$ & $371.9 \mathrm{a}-\mathrm{d}$ & $160.4 \mathrm{ab}$ & $988.7 \mathrm{a}-\mathrm{c}$ \\
\hline 2. Sintético interfamiliar & 162.7 e-1 & $207.4 \mathrm{c}-\mathrm{g}$ & $156.7 \mathrm{ab}$ & 748.5 b-j \\
\hline 3. Familia 130 & $251.9 \mathrm{c}-\mathrm{h}$ & 333.9 a-e & $128.0 \mathrm{ab}$ & 713.9 b-m \\
\hline 4. Población 8 & 390.0 a-d & $266.6 \mathrm{a}-\mathrm{g}$ & $191.1 \mathrm{ab}$ & $847.7 \mathrm{a}-\mathrm{g}$ \\
\hline 5. Población 5 & $438.5 \mathrm{a}-\mathrm{c}$ & $381.7 \mathrm{a}-\mathrm{c}$ & $205.0 \mathrm{ab}$ & $1025.1 \mathrm{ab}$ \\
\hline 6. Población 3 & $516.5 \mathrm{a}$ & $428.7 \mathrm{ab}$ & $157.4 \mathrm{ab}$ & $1102.5 \mathrm{a}$ \\
\hline 7. Población 2 & 311.3 b-e & 388.8 a-c & $207.1 \mathrm{ab}$ & 907.3 a-e \\
\hline 8. Sintético intervarietal & $238.8 \mathrm{~d}-\mathrm{j}$ & $279.3 \mathrm{a}-\mathrm{g}$ & $257.4 \mathrm{ab}$ & $775.5 a-j$ \\
\hline 9. Manzano SM2R & 286.7 b-f & $432.7 \mathrm{a}$ & $252.9 \mathrm{ab}$ & $972.4 \mathrm{a}-\mathrm{d}$ \\
\hline 10. Puebla SM1 & $75.3 \mathrm{~h}-1$ & $204.4 \mathrm{c}-\mathrm{g}$ & $258.9 \mathrm{ab}$ & $538.6 \mathrm{~g}-\mathrm{o}$ \\
\hline 11. Esmeralda & $62.3 \mathrm{~h}-1$ & $133.5 \mathrm{e}-\mathrm{g}$ & $194.3 \mathrm{ab}$ & $390.0 \mathrm{~m}-\mathrm{o}$ \\
\hline 12. Verde 3000 & 27.91 & $140.4 \mathrm{e}-\mathrm{g}$ & $225.1 \mathrm{ab}$ & 599.3 e-o \\
\hline 13. Verde supremo & $75.1 \mathrm{~h}-1$ & 227.0 b-g & $217.0 \mathrm{ab}$ & 519.2 g-o \\
\hline 14. Yoreme & $71.5 \mathrm{~h}-1$ & $143.3 \mathrm{e}-\mathrm{g}$ & $149.6 \mathrm{ab}$ & 364.4 no \\
\hline 15. Súper morado & $179.6 \mathrm{e}-1$ & $294.0 \mathrm{a}-\mathrm{g}$ & $184.6 \mathrm{ab}$ & $658.1 \mathrm{c}-\mathrm{n}$ \\
\hline 16. Súper cerro gordo & $156.5 \mathrm{e}-1$ & 275.7 a-g & $122.1 \mathrm{~b}$ & $554.2 \mathrm{f}-\mathrm{o}$ \\
\hline 17. Tamazula SM2 & $229.8 \mathrm{~d}-\mathrm{k}$ & 307.7 a-f & $120.0 \mathrm{~b}$ & $657.5 \mathrm{c}-\mathrm{n}$ \\
\hline 18. San Mateo & $77.4 \mathrm{~g}-1$ & $120.6 \mathrm{fg}$ & $255.9 \mathrm{ab}$ & $453.8 \mathrm{j}$-o \\
\hline 19. Diamante & $248.1 \mathrm{c}-\mathrm{i}$ & 318.9 a-f & $220.1 \mathrm{ab}$ & $787.2 \mathrm{a}-\mathrm{i}$ \\
\hline 20. Puebla SM2 & $65.0 \mathrm{~h}-\mathrm{l}$ & 150.9 e-g & $232.8 \mathrm{ab}$ & $448.7 \mathrm{j}$-o \\
\hline 21. Tamazula SM2R & $268.1 \mathrm{~b}-\mathrm{g}$ & $267.7 \mathrm{a}-\mathrm{g}$ & $198.6 \mathrm{ab}$ & $734.4 \mathrm{~b}-\mathrm{k}$ \\
\hline 22. Tamazula SM3 & $252.6 \mathrm{c}-\mathrm{h}$ & 327.6 a-e & $144.9 \mathrm{ab}$ & $725.1 \mathrm{~b}-1$ \\
\hline 23. 19 JAL.74 & $107.8 \mathrm{f}-1$ & $226.3 \mathrm{c}-\mathrm{g}$ & $211.6 \mathrm{ab}$ & $545.7 \mathrm{f}-\mathrm{o}$ \\
\hline 24. 185 JAL. 68 & $176.5 \mathrm{e}-1$ & $281.1 \mathrm{a}-\mathrm{g}$ & $160.3 \mathrm{ab}$ & 617.9 e-n \\
\hline 25. 12 GUSA02 & $58.2 \mathrm{i}-1$ & $177.3 \mathrm{~d}-\mathrm{g}$ & $239.4 \mathrm{ab}$ & 475.0 i-o \\
\hline 26. Puebla verde & $206.2 \mathrm{~d}-1$ & 316.6 a-f & $252.0 \mathrm{ab}$ & $774.7 a-j$ \\
\hline 27. 128 BCN01ELSA & $230.3 \mathrm{~d}-\mathrm{k}$ & 328.9 a-e & $203.0 \mathrm{ab}$ & $762.2 \mathrm{~b}-\mathrm{j}$ \\
\hline 28. 124 USA 03 & 322.0 b-e & $384.5 \mathrm{a}-\mathrm{c}$ & $131.7 \mathrm{ab}$ & 838.3 a-h \\
\hline 29. 127 USA 03 & $159.6 \mathrm{e}-1$ & 306.9 a-f & $180.4 \mathrm{ab}$ & $646.8 \mathrm{~d}-\mathrm{n}$ \\
\hline 30. $129 \mathrm{BCN} 02$ & 306.8 b-e & 326.5 a-e & $204.2 \mathrm{ab}$ & 837.5 a-h \\
\hline 31. Mahune & 146.6 e-1 & $318.2 \mathrm{a}-\mathrm{f}$ & $181.1 \mathrm{ab}$ & 645.9 d-n \\
\hline 32. Cerro gordo & $87.6 \mathrm{~g}-1$ & $164.5 \mathrm{e}-\mathrm{g}$ & $236.1 \mathrm{ab}$ & 488.1 i-o \\
\hline 33. San Román & 36.81 & $104.5 \mathrm{~g}$ & $132.0 \mathrm{ab}$ & $273.4 \mathrm{o}$ \\
\hline 34. Rendidora mejorada & $44.6 \mathrm{kl}$ & $136.5 \mathrm{e}-\mathrm{g}$ & $233.6 \mathrm{ab}$ & $414.7 \mathrm{k}-\mathrm{o}$ \\
\hline 35. Salamanca & $50.4 \mathrm{j}-1$ & $156.0 \mathrm{e}-\mathrm{g}$ & $194.9 \mathrm{ab}$ & $401.31-0$ \\
\hline 36. Cerro gordo AN & $59.1 \mathrm{i}-1$ & $137.7 \mathrm{e}-\mathrm{g}$ & $266.2 \mathrm{ab}$ & 462.9 i-o \\
\hline 37. Boyeros & $252.4 \mathrm{c}-\mathrm{h}$ & 304.9 a-g & $316.1 \mathrm{a}$ & $873.4 \mathrm{a}-\mathrm{f}$ \\
\hline 38. Tecamachalco & $101.9 \mathrm{f}-1$ & $232.6 \mathrm{a}-\mathrm{g}$ & $177.8 \mathrm{ab}$ & $512.4 \mathrm{~h}-\mathrm{o}$ \\
\hline 39. Divino & 60.6 i-1 & $296.1 \mathrm{a}-\mathrm{g}$ & $229.7 \mathrm{ab}$ & 586.5 e-o \\
\hline 40. 126 USA 02 & $66.6 \mathrm{~h}-1$ & $214.6 \mathrm{c}-\mathrm{g}$ & $164.0 \mathrm{ab}$ & $445.2 \mathrm{j}-\mathrm{o}$ \\
\hline DMSH & 190.9 & 201.7 & 193.2 & 332.3 \\
\hline Promedio & 182.9 & 260.4 & 198.8 & 652.8 \\
\hline
\end{tabular}

$\mathrm{DMSH}$ = diferencia mínima significativa honesta. ${ }^{\dagger}$ Medias con la misma letra en una columna no difieren estadísticamente (Tukey, 0.05); sólo se incluyen las letras inicial y final de cada media unidas por un guión. 
Cuadro 5. Medias de peso (g) por fruto promedio de cortes (PPPF) de 40 variedades de tomate de cáscara cultivadas en cuatro sistemas producción [riego rodado (RR), riego por goteo sin acolchado (RGSA), riego por goteo con acolchado (RGCA) e hidroponía bajo invernadero (HI)]. Chapingo, México.

\begin{tabular}{|c|c|c|c|c|}
\hline Variedad & $\mathrm{RR}$ & RGSA & RGCA & HI \\
\hline 1. CHF1-Chapingo & $28.8 \mathrm{~b}-\mathrm{h}^{\dagger}$ & $19.5 \mathrm{c}-\mathrm{g}$ & 30.6 e-m & $29.3 \mathrm{a}-\mathrm{g}$ \\
\hline 2. Sintético interfamiliar & - & 19.7 c-g & $33.6 \mathrm{~d}-\mathrm{j}$ & $28.1 \mathrm{a}-\mathrm{g}$ \\
\hline 3. Familia 130 & $24.6 \mathrm{e}-\mathrm{j}$ & $19.4 \mathrm{c}-\mathrm{g}$ & $33.7 \mathrm{~d}-\mathrm{i}$ & $32.4 \mathrm{a}-\mathrm{g}$ \\
\hline 4. Población 8 & 31.9 b-f & $20.5 \mathrm{c}-\mathrm{g}$ & 30.8 e-m & 35.9 a-g \\
\hline 5. Población 5 & $26.8 \mathrm{~d}-\mathrm{j}$ & $20.7 \mathrm{c}-\mathrm{g}$ & $33.6 \mathrm{~d}-\mathrm{j}$ & 35.9 a-g \\
\hline 6. Población 3 & $27.9 c-i$ & $18.2 \mathrm{c}-\mathrm{g}$ & $32.4 \mathrm{e}-\mathrm{k}$ & $29.2 \mathrm{a}-\mathrm{g}$ \\
\hline 7. Población 2 & $29.5 \mathrm{~b}-\mathrm{g}$ & $20.3 \mathrm{c}-\mathrm{g}$ & $33.7 \mathrm{~d}-\mathrm{i}$ & $32.7 \mathrm{a}-\mathrm{g}$ \\
\hline 8. Sintético intervarietal & 36.2 a-e & $25.7 b-f$ & $45.1 \mathrm{~b}-\mathrm{e}$ & $42.3 \mathrm{a}-\mathrm{g}$ \\
\hline 9. Manzano SM2R & $27.2 \mathrm{~d}-\mathrm{i}$ & $23.9 \mathrm{c}-\mathrm{g}$ & $35.2 \mathrm{c}-\mathrm{h}$ & $38.4 \mathrm{a}-\mathrm{g}$ \\
\hline 10. Puebla SM1 & $47.2 \mathrm{a}$ & $36.8 \mathrm{ab}$ & 48.2 a-d & $50.1 \mathrm{a}-\mathrm{d}$ \\
\hline 11. Esmeralda & $20.1 \mathrm{f}-\mathrm{j}$ & $21.2 \mathrm{c}-\mathrm{g}$ & $25.5 \mathrm{~g}-\mathrm{m}$ & $22.3 \mathrm{~d}-\mathrm{g}$ \\
\hline 12. Verde 3000 & - & $17.5 \mathrm{~d}-\mathrm{g}$ & $26.1 \mathrm{~g}-\mathrm{m}$ & $17.7 \mathrm{~g}$ \\
\hline 13. Verde supremo & $16.8 \mathrm{~g}-\mathrm{j}$ & $26.6 \mathrm{~b}-\mathrm{e}$ & $24.9 \mathrm{~g}-\mathrm{m}$ & $23.1 \mathrm{~d}-\mathrm{g}$ \\
\hline 14. Yoreme & $20.7 \mathrm{f}-\mathrm{j}$ & $19.0 \mathrm{c}-\mathrm{g}$ & $26.0 \mathrm{~g}-\mathrm{m}$ & $21.2 \mathrm{e}-\mathrm{g}$ \\
\hline 15. Súper morado & $15.4 \mathrm{ij}$ & 14.6 e-g & $19.1 \mathrm{i}-\mathrm{m}$ & $22.7 \mathrm{~d}-\mathrm{g}$ \\
\hline 16. Súper cerro gordo & $15.5 \mathrm{ij}$ & $13.5 \mathrm{fg}$ & $16.2 \mathrm{~m}$ & $17.8 \mathrm{~g}$ \\
\hline 17. Tamazula SM2 & $15.2 \mathrm{ij}$ & $13.4 \mathrm{fg}$ & $17.0 \mathrm{~lm}$ & $18.1 \mathrm{~g}$ \\
\hline 18. San Mateo & $41.6 \mathrm{ab}$ & $23.7 \mathrm{c}-\mathrm{g}$ & 60.9 a & $52.1 \mathrm{a}-\mathrm{c}$ \\
\hline 19. Diamante & 38.6 a-d & $30.8 \mathrm{a}-\mathrm{c}$ & 44.0 b-e & 49.0 a-e \\
\hline 20. Puebla SM2 & 40.8 a-c & $40.3 \mathrm{a}$ & $52.6 \mathrm{ab}$ & $54.6 \mathrm{ab}$ \\
\hline 21. Tamazula SM2R & $16.3 \mathrm{~h}-\mathrm{j}$ & $13.2 \mathrm{fg}$ & $18.7 \mathrm{j}-\mathrm{m}$ & $19.2 \mathrm{fg}$ \\
\hline 22. Tamazula SM3 & $15.7 \mathrm{ij}$ & $12.6 \mathrm{~g}$ & $21.6 \mathrm{~h}-\mathrm{m}$ & $16.3 \mathrm{~g}$ \\
\hline 23. 19 JAL.74 & $16.7 \mathrm{~g}-\mathrm{j}$ & $13.8 \mathrm{e}-\mathrm{g}$ & $17.7 \mathrm{k}-\mathrm{m}$ & $19.0 \mathrm{fg}$ \\
\hline 24. 185 JAL. 68 & $14.1 \mathrm{j}$ & $13.6 \mathrm{fg}$ & $17.1 \mathrm{~lm}$ & $15.8 \mathrm{~g}$ \\
\hline 25. 12 GUSA02 & $19.8 \mathrm{f}-\mathrm{j}$ & $18.2 \mathrm{c}-\mathrm{g}$ & $23.1 \mathrm{~g}-\mathrm{m}$ & $21.9 \mathrm{~d}-\mathrm{g}$ \\
\hline 26. Puebla verde & $25.1 \mathrm{e}-\mathrm{j}$ & $21.6 \mathrm{c}-\mathrm{g}$ & $28.0 \mathrm{f}-\mathrm{m}$ & $33.1 \mathrm{a}-\mathrm{g}$ \\
\hline 27. 128 BCN01ELSA & $30.3 \mathrm{~b}-\mathrm{f}$ & $21.2 \mathrm{c}-\mathrm{g}$ & $31.3 \mathrm{e}-1$ & $34.8 \mathrm{a}-\mathrm{g}$ \\
\hline 28. 124 USA 03 & $29.5 \mathrm{~b}-\mathrm{g}$ & $20.2 \mathrm{c}-\mathrm{g}$ & $27.3 \mathrm{f}-\mathrm{m}$ & 30.9 a-g \\
\hline 29. 127 USA 03 & $20.00 \mathrm{f}-\mathrm{j}$ & $18.4 \mathrm{c}-\mathrm{g}$ & $28.4 \mathrm{f}-\mathrm{m}$ & $29.3 \mathrm{a}-\mathrm{g}$ \\
\hline 30. $129 \mathrm{BCN} 02$ & $28.2 \mathrm{c}-\mathrm{i}$ & $21.8 \mathrm{c}-\mathrm{g}$ & $32.1 \mathrm{e}-\mathrm{k}$ & $39.9 \mathrm{a}-\mathrm{g}$ \\
\hline 31. Mahune & $46.4 \mathrm{a}$ & 28.7 a-d & $49.3 \mathrm{a}-\mathrm{c}$ & 55.7 a \\
\hline 32. Cerro gordo & $23.0 \mathrm{f}-\mathrm{j}$ & $21.6 \mathrm{c}-\mathrm{g}$ & 27.9 f-m & $26.3 \mathrm{~b}-\mathrm{g}$ \\
\hline 33. San Román & $20.8 \mathrm{f}-\mathrm{j}$ & $21.8 \mathrm{c}-\mathrm{g}$ & $31.7 \mathrm{e}-\mathrm{k}$ & $27.4 \mathrm{a}-\mathrm{g}$ \\
\hline 34. Rendidora mejorada & $22.3 \mathrm{f}-\mathrm{j}$ & $20.2 \mathrm{c}-\mathrm{g}$ & $27.6 \mathrm{f}-\mathrm{m}$ & $22.6 \mathrm{~d}-\mathrm{g}$ \\
\hline 35. Salamanca & $25.7 \mathrm{~d}-\mathrm{j}$ & $20.2 \mathrm{c}-\mathrm{g}$ & $25.3 \mathrm{~g}-\mathrm{m}$ & $26.1 \mathrm{~b}-\mathrm{g}$ \\
\hline 36. Cerro gordo AN & $26.3 \mathrm{~d}-\mathrm{j}$ & $17.8 \mathrm{~d}-\mathrm{g}$ & $26.0 \mathrm{~g}-\mathrm{m}$ & $24.7 \quad c-g$ \\
\hline 37. Boyeros & 36.8 a-e & $28.1 \mathrm{a}-\mathrm{d}$ & $41.2 \mathrm{~b}-\mathrm{f}$ & 43.3 a-g \\
\hline 38. Tecamachalco & $41.6 \mathrm{ab}$ & $24.3 \mathrm{~b}-\mathrm{f}$ & $51.5 \mathrm{ab}$ & $47.7 \mathrm{a}-\mathrm{f}$ \\
\hline 39. Divino & $20.7 \mathrm{f}-\mathrm{j}$ & 28.7 a-d & $37.9 \mathrm{~b}-\mathrm{g}$ & $35.6 \mathrm{a}-\mathrm{g}$ \\
\hline 40. 126 USA 02 & $0.1 \mathrm{k}$ & $18.1 \mathrm{c}-\mathrm{g}$ & $23.1 \mathrm{~g}-\mathrm{m}$ & $25.0 \mathrm{c}-\mathrm{g}$ \\
\hline DMSH & 13.1 & 12.9 & 15.0 & 28.8 \\
\hline Promedio & 26.4 & 21.2 & 31.4 & 31.2 \\
\hline
\end{tabular}

DMSH = diferencia mínima significativa honesta. ${ }^{\dagger}$ Medias con la misma letra en una columna, no difieren estadísticamente (Tukey, 0.05); sólo se incluyen las letras inicial y final de cada media unidas por un guión. 
Cuadro 6. Medias de rendimiento total (g) por planta (RTP) de 40 variedades de tomate de cáscara cultivadas en cuatro sistemas producción [riego rodado (RR), riego por goteo sin acolchado (RGSA), riego por goteo con acolchado (RGCA) e hidroponía bajo invernadero (HI)]. Chapingo, México.

\begin{tabular}{|c|c|c|c|c|}
\hline Variedad & $\mathrm{RR}$ & RGSA & RGCA & HI \\
\hline 1. CHF1-Chapingo & $807.1{\mathrm{a}-\mathrm{f}^{\dagger}}^{\dagger}$ & $757.9 \mathrm{ab}$ & 1447.8 a-c & 942.0 a-e \\
\hline 2. Sintético interfamiliar & - & $460.0 \mathrm{a}-\mathrm{c}$ & 683.8 b-e & 400.3 b-g \\
\hline 3. Familia 130 & $591.8 \mathrm{~b}-\mathrm{j}$ & 486.3 a-c & 1127.8 a-e & $649.5 \mathrm{a}-\mathrm{g}$ \\
\hline 4. Población 8 & $1045.9 \mathrm{ab}$ & $441.4 \mathrm{a}-\mathrm{c}$ & 1017.6 a-e & 886.0 a-g \\
\hline 5. Población 5 & $1009.6 \mathrm{a}-\mathrm{c}$ & $548.5 \mathrm{a}-\mathrm{c}$ & 1541.9 a & $1000.5 \mathrm{a}-\mathrm{d}$ \\
\hline 6. Población 3 & $1122.0 \mathrm{a}$ & $793.5 \mathrm{a}$ & $1486.2 \mathrm{ab}$ & $1008.3 \mathrm{a}-\mathrm{c}$ \\
\hline 7. Población 2 & 940.7 a-d & $474.7 \mathrm{a}-\mathrm{c}$ & 1203.1 a-e & $1010.6 \mathrm{a}-\mathrm{c}$ \\
\hline 8. Sintético intervarietal & $603.8 \mathrm{a}-\mathrm{j}$ & $597.2 \mathrm{a}-\mathrm{c}$ & 1156.7 a-e & $744.1 \mathrm{a}-\mathrm{g}$ \\
\hline 9. Manzano SM2R & 814.6 a-f & $623.2 \mathrm{a}-\mathrm{c}$ & 1390.8 a-d & $1060.8 \mathrm{ab}$ \\
\hline 10. Puebla SM1 & $509.6 \mathrm{c}-\mathrm{k}$ & $348.2 \mathrm{a}-\mathrm{c}$ & 940.0 a-e & $356.7 \mathrm{c}-\mathrm{g}$ \\
\hline 11. Esmeralda & $179.9 \mathrm{jk}$ & $357.4 \mathrm{a}-\mathrm{c}$ & 767.8 a-e & $260.1 \mathrm{e}-\mathrm{g}$ \\
\hline 12. Verde 3000 & 301.9 f-k & $308.2 \mathrm{a}-\mathrm{c}$ & $550.5 \mathrm{de}$ & $304.0 \mathrm{~d}-\mathrm{g}$ \\
\hline 13. Verde supremo & $240.2 \mathrm{i}-\mathrm{k}$ & $390.7 \mathrm{a}-\mathrm{c}$ & 836.5 a-e & $547.6 \mathrm{a}-\mathrm{g}$ \\
\hline 14. Yoreme & 381.0 e-k & 265.9 a-c & $633.8 c-e$ & $317.5 \mathrm{c}-\mathrm{g}$ \\
\hline 15. Súper morado & $281.8 \mathrm{~g}-\mathrm{k}$ & $320.3 \mathrm{a}-\mathrm{c}$ & 1120.0 a-e & $811.3 \mathrm{a}-\mathrm{g}$ \\
\hline 16. Súper cerro gordo & $507.1 \mathrm{c}-\mathrm{k}$ & $339.5 \mathrm{a}-\mathrm{c}$ & 872.7 a-e & 722.8 a-g \\
\hline 17. Tamazula SM2 & $535.0 \mathrm{~b}-\mathrm{j}$ & $324.3 \mathrm{a}-\mathrm{c}$ & 1128.4 a-e & $670.0 \mathrm{a}-\mathrm{g}$ \\
\hline 18. San Mateo & 837.4 a-e & $209.6 \mathrm{c}$ & 715.9 a-e & $354.6 \mathrm{c}-\mathrm{g}$ \\
\hline 19. Diamante & $480.7 \mathrm{~d}-\mathrm{k}$ & $512.5 \mathrm{a}-\mathrm{c}$ & 1046.6 a-e & $752.1 \mathrm{a}-\mathrm{g}$ \\
\hline 20. Puebla SM2 & - & $352.1 \mathrm{a}-\mathrm{c}$ & $616.9 \mathrm{c}-\mathrm{e}$ & $345.1 \mathrm{c}-\mathrm{g}$ \\
\hline 21. Tamazula SM2R & $458.2 \mathrm{~d}-\mathrm{k}$ & $423.0 \mathrm{a}-\mathrm{c}$ & 1280.1 a-e & $776.4 \mathrm{a}-\mathrm{g}$ \\
\hline 22. Tamazula SM3 & $500.8 \mathrm{c}-\mathrm{k}$ & $386.2 \mathrm{a}-\mathrm{c}$ & 1163.2 a-e & 850.3 a-g \\
\hline 23. 19 JAL.74 & $379.5 \mathrm{e}-\mathrm{k}$ & $317.6 \mathrm{a}-\mathrm{c}$ & 936.5 a-e & $549.5 \mathrm{a}-\mathrm{g}$ \\
\hline 24. 185 JAL. 68 & $472.4 \mathrm{~d}-\mathrm{k}$ & $377.1 \mathrm{a}-\mathrm{c}$ & 958.4 a-e & $663.6 \mathrm{a}-\mathrm{g}$ \\
\hline 25. 12 GUSA02 & $253.6 \mathrm{~h}-\mathrm{k}$ & $364.9 \mathrm{a}-\mathrm{c}$ & 703.7 a-e & $577.5 \mathrm{a}-\mathrm{g}$ \\
\hline 26. Puebla verde & 772.9 a-h & $579.2 \mathrm{a}-\mathrm{c}$ & 1108.3 a-e & $638.6 \mathrm{a}-\mathrm{g}$ \\
\hline 27. 128 BCN01ELSA & 794.5 a-g & $563.9 \mathrm{a}-\mathrm{c}$ & 859.5 a-e & 830.8 a-g \\
\hline 28. 124 USA 03 & 728.9 a-i & $613.5 \mathrm{a}-\mathrm{c}$ & 861.3 a-e & $1149.2 \mathrm{a}$ \\
\hline 29. 127 USA 03 & $478.8 \mathrm{~d}-\mathrm{k}$ & $672.1 \mathrm{a}-\mathrm{c}$ & 1025.8 a-e & 410.6 b-g \\
\hline 30. $129 \mathrm{BCN} 02$ & $682.1 \mathrm{a}-\mathrm{j}$ & $532.0 \mathrm{a}-\mathrm{c}$ & 1214.5 a-e & 921.5 a-e \\
\hline 31. 'Mahune' & $674.7 a-j$ & $627.2 \mathrm{a}-\mathrm{c}$ & 634.7 c-e & $647.2 \mathrm{a}-\mathrm{g}$ \\
\hline 32. Cerro gordo & $371.1 \mathrm{e}-\mathrm{k}$ & $311.5 \mathrm{a}-\mathrm{c}$ & 975.2 a-e & 294.7 e-g \\
\hline 33. San Román & $160.3 \mathrm{jk}$ & $249.3 \mathrm{bc}$ & $483.8 \mathrm{e}$ & $235.5 \mathrm{fg}$ \\
\hline 34. Rendidora mejorada & $297.1 \mathrm{f}-\mathrm{k}$ & 329.5 a-c & 796.6 a-e & $200.0 \mathrm{~g}$ \\
\hline 35. Salamanca & 332.3 e-k & $227.9 \mathrm{bc}$ & 692.7 b-e & $362.5 \mathrm{c}-\mathrm{g}$ \\
\hline 36. Cerro gordo AN & $458.1 \mathrm{~d}-\mathrm{k}$ & 281.9 a-c & 787.4 a-e & $324.3 \mathrm{c}-\mathrm{g}$ \\
\hline 37. Boyeros & 958.0 a-d & 566.7 a-c & 1081.1 a-e & 887.9 a-g \\
\hline 38. Tecamachalco & $634.0 a-j$ & $382.4 \mathrm{a}-\mathrm{c}$ & $595.1 \mathrm{de}$ & 437.9 b-g \\
\hline 39. Divino & $445.3 \mathrm{~d}-\mathrm{k}$ & $577.5 \mathrm{a}-\mathrm{c}$ & 827.4 a-e & $344.2 \mathrm{c}-\mathrm{g}$ \\
\hline 40. 126 USA 02 & $0.6 \mathrm{k}$ & $317.7 \mathrm{a}-\mathrm{c}$ & $643.6 \mathrm{c}-\mathrm{e}$ & $374.0 \mathrm{~b}-\mathrm{g}$ \\
\hline DMSH & 524.9 & 542.6 & 841.5 & 699.9 \\
\hline Promedio & 559.0 & 440.3 & 947.8 & 615.5 \\
\hline
\end{tabular}

$\mathrm{DMSH}=$ diferencia mínima significativa honesta. ${ }^{\dagger}$ Medias con la misma letra en una columna, no difieren estadísticamente (Tukey, 0.05 ); sólo se incluyen las letras inicial y final de cada media unidas por un guión. 
(Cuadro 6). En el sistema de producción de riego por goteo sin acolchado las dos mejores variedades fueron Población 3 y CHF1-Chapingo, aunque sólo superaron estadísticamente a tres y una variedades, respectivamente. En el sistema de producción de riego por goteo con acolchado, de las 35 variedades de mayor rendimiento la Población 5 superó a nueve variedades, y la Población 3 a 7. Las mejores variedades para el sistema de producción de hidroponía bajo invernadero fueron 124 USA 03, Manzano SM2R, Población 2, Población 3 y Población 5, pero la primera sólo superó a las 16 variedades de menor RTP. Destaca nuevamente que las variedades de mayor rendimiento en cada sistema de producción y en promedio de ellos son de la raza Rendidora.

\section{Variedades sobresalientes por raza}

Al considerar el $10 \%$ mejor de las variedades evaluadas, las mejores cuatro para PPPF fueron Puebla SM2, Puebla SM1, Mahune y San Mateo, que sobresalieron en al menos dos sistemas de producción y fueron las mejores en promedio de los cuatro sistemas estudiados (Cuadros 3 y 5 ). Todas las variedades con mayor peso de fruto $(10 \%$ mejor por sistema y en promedio de ellos) son de la raza Puebla, excepto Diamante que es un híbrido entre las razas Rendidora y Puebla. Lo anterior permite confirmar que el germoplasma de la raza Puebla posee mayor tamaño de fruto, aspecto importante para la exportación cuyo mercado demanda frutos grandes.

Para rendimiento por planta (Cuadros 4 y 6) las mejores variedades fueron Población 3, Población 5, CHF1-Chapingo y Manzano SM2R, que se ubicaron en al menos tres ocasiones en el grupo de alto rendimiento ( $10 \%$ mejor por sistema y en promedio de ellos). Con excepción de la variedad Manzano SM2R, el resto pertenecen a la raza Rendidora que es de alta productividad.

\section{CONCLUSIONES}

El mejor sistema de producción en cuanto a rendimiento y tamaño de fruto fue el de campo abierto con riego por goteo y acolchado, seguido de hidroponía bajo invernadero. Las 40 variedades evaluadas difirieron en su comportamiento en los cuatro sistemas de producción. Las variedades que lograron el mayor rendimiento de fruto, en promedio de los cuatro sistemas de producción, fueron Población 3, Población 5, CHF1-Chapingo y Manzano SM2R, con frutos de tamaño mediano; en tanto que las variedades de mayor tamaño de fruto fueron Puebla SM2, Puebla SM1, Mahune y San Mateo, aunque presentaron bajo rendimiento. En general, las variedades de mayor rendimiento son de la raza Rendidora y las de mayor tamaño de fruto de la raza Puebla.
El rendimiento en el primer corte es fundamental para obtener un alto rendimiento total, pues en general las variedades más rendidoras al inicio mantuvieron esta característica hasta el final. Para cada sistema de producción (riego rodado, riego por goteo con y sin acolchado plástico e hidroponía bajo invernadero), las variedades de mayor rendimiento fueron Población 3, Población 5, Población 3 y 124 USA 03, respectivamente; en tanto que las de mayor tamaño de fruto fueron Puebla SM1, San Mateo, Puebla SM2 y Mahune, respectivamente.

\section{BIBLIOGRAFÍA}

Cachón A. L. E., H. Nery G. y H. E. Cuanalo C (1974) Los Suelos del Área de Influencia de Chapingo. ENA. Colegio de Postgraduados. Chapingo, México. $79 \mathrm{p}$.

Castro B. R., P. Sánchez-García, A. Peña-Lomelí, G. Alcántar-González, G. A. Baca-Castillo y R. M. López-Romero (2000) Nitratos en el extracto celular de pecíolos y tallo de tomate de cáscara (Physalys ixocarpa Brot.) y su relación con el rendimiento en Chapingo, México. Revista Chapingo Serie Horticultura 6:33-38.

Cruz-Álvarez O., M. T. Martínez-Damián, J. E. Rodríguez-Pérez, M. T. Colinas-León y E. C. Moreno-Pérez (2012) Conservación poscosecha de tomate de cáscara (Physalis ixocarpa Brot. ex Horm.) con y sin cáliz. Revista Chapingo Serie Horticultura 18:333-344.

Csizirtszky A. A., D. I. Schuster and J. B. Kring (1995) Color mulches influence yield and insect pest populations in tomatoes. Journal of the American Society for Horticultural Science 120:778-784.

Peña L. A., N. Magaña L., S. Montes H., J. Sánchez M., J. F. Santiaguillo H., O. Grimaldo J. y A. Contreras R. (2011) Manual gráfico para la descripción varietal de tomate de cáscara (Physalis ixocarpa Brot. ex Horm.). SNICS-SAGARPA, Universidad Autónoma Chapingo, Chapingo, Estado de México, México. 87 p.

Peña L. A. y F. Márquez S. (1990) Mejoramiento genético del tomate de cáscara (Physalys ixocarpa Brot.). Revista Chapingo 71-72:84-88.

Peña L. A., J. D. Molina G., F. Márquez S., J. Sahagún C., J. Ortiz C. y T. Cervantes S. (1999) Heterosis intravarietal en tomate de cáscara (Physalis ixocarpa Brot.). Revista Fitotecnia Mexicana 22:199-213.

Peña L. A., J. D. Molina G., J. Sahagún C., J. Ortiz C., F. Márquez S., T. Cervantes S. y J. F. Santiaguillo H. (2008) Parámetros genéticos de la variedad CHF1 Chapingo de tomate de cáscara (Physalis ixocarpa Brot.). Revista Chapingo Serie Horticultura 14:5-11.

Peña L. A. y J. F. Santiaguillo H. (1999) Variabilidad Genética de Tomate de Cáscara en México. Boletín \# 2. Departamento de Fitotecnia. Universidad Autónoma Chapingo. Chapingo, México. 26 p.

Pérez G. M. y R. Castro B. (1999) Guía para la Producción Intensiva de Jitomate en Invernadero. Boletín \# 3. Departamento de Fitotecnia. Universidad Autónoma Chapingo. Chapingo, México. 58 p.

Pérez G. M., F. Márquez S. y A. Peña L. (1997) Mejoramiento Genético de Hortalizas. Universidad Autónoma Chapingo. Chapingo México. pp:217-229.

Sahagún C. J. (1993) Funcionalidad de cuatro modelos para las evaluaciones genotípicas en series de experimentos. Revista Fitotecnia Mexicana 16:161-171.

SNIIM, Servicio Nacional de Información e Integración de Mercados (2012) Anuarios Estadísticos de Mercados Nacionales: frutas y hortalizas. Disponible en: http://www.economia-sniim.gob.mx (Octubre 2012).

SIAP-SAGARPA, Sistema de Información Agroalimentaria y Pesquera-Secretaría de Agricultura, Ganadería, Desarrollo Rural, Pesca y Alimentación (2010) Cierre de la producción agrícola por cultivo. México. Disponible en: http://www.siap.gob.mx (Diciembre 2011).

Santiaguillo H. J. F., T. Cervantes S. y A. Peña L. (2004) Selección para rendimiento y calidad de fruto de cruzas planta $\mathrm{x}$ planta entre 
variedades de tomate de cáscara. Revista Fitotecnia Mexicana 27:85-91.

Santiaguillo H. J. F., O. Vargas P., O. Grimaldo J., N. Magaña L., F. J. Caro V., A. Peña L. y J. Sánchez M. (2012) Diagnóstico del Tomate de Cáscara. Universidad Autónoma Chapingo. Chapingo,
México. $46 \mathrm{p}$

Soldevilla C. S., A. Peña L., F. Solís M., T. Vásquez R. y M. T. Colinas L. (2002) Aplicación al Suelo de $\mathrm{CO}_{2}$, uso de acolchados plásticos y sistemas de manejo en tomate de cáscara (Physalis ixocarpa Brot.). Revista Chapingo Serie Horticultura 8:25-38. 